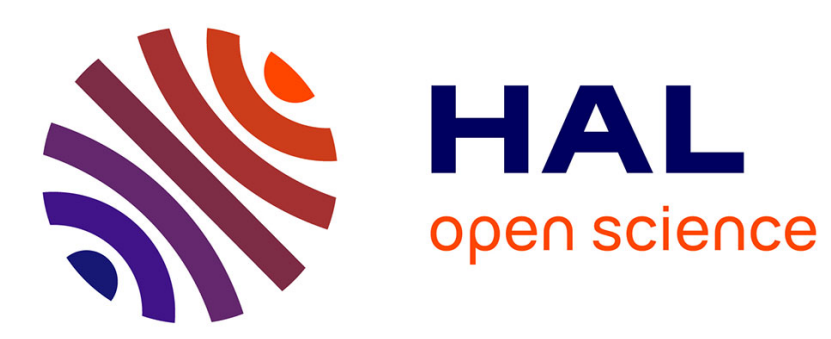

\title{
Amour chevaleresque, amour galant et discours politiques de l'amour dans Les Plaisirs de l'île enchantée(1664)
}

Marine Roussillon

\section{- To cite this version:}

Marine Roussillon. Amour chevaleresque, amour galant et discours politiques de l'amour dans Les Plaisirs de l'île enchantée(1664). Littératures classiques, 2009, Les discours artistiques de l'amour à l'âge classique, 2 (69), pp.65-78. 10.3917/licla.069.0065 . halshs-01487625

\section{HAL Id: halshs-01487625 \\ https://shs.hal.science/halshs-01487625}

Submitted on 12 Mar 2017

HAL is a multi-disciplinary open access archive for the deposit and dissemination of scientific research documents, whether they are published or not. The documents may come from teaching and research institutions in France or abroad, or from public or private research centers.
L'archive ouverte pluridisciplinaire HAL, est destinée au dépôt et à la diffusion de documents scientifiques de niveau recherche, publiés ou non, émanant des établissements d'enseignement et de recherche français ou étrangers, des laboratoires publics ou privés. 


\section{AMOUR CHEVALERESQUE, AMOUR GALANT ET DISCOURS POLITIQUES DE L'AMOUR DANS LES PLAISIRS DE L'ÎLE ENCHANTÉE(1664) \\ Marine Roussillon}

Armand Colin | «Littératures classiques »

2009/2 N 69 | pages 65 à 78

ISSN 0992-5279

ISBN 9782908728606

Article disponible en ligne à l'adresse :

http://www.cairn.info/revue-litteratures-classiques1-2009-2-page-65.htm

\section{Pour citer cet article :}

Marine Roussillon, "Amour chevaleresque, amour galant et discours politiques de l'amour dans Les Plaisirs de l'île enchantée(1664) 》, Littératures classiques 2009/2 $\left(\mathrm{N}^{\circ} 69\right)$, p. 65-78.

DOI 10.3917/licla.069.0065

Distribution électronique Cairn.info pour Armand Colin.

(C) Armand Colin. Tous droits réservés pour tous pays.

La reproduction ou représentation de cet article, notamment par photocopie, n'est autorisée que dans les limites des conditions générales d'utilisation du site ou, le cas échéant, des conditions générales de la licence souscrite par votre établissement. Toute autre reproduction ou représentation, en tout ou partie, sous quelque forme et de quelque manière que ce soit, est interdite sauf accord préalable et écrit de l'éditeur, en dehors des cas prévus par la législation en vigueur en France. Il est précisé que son stockage dans une base de données est également interdit. 
Marine Roussillon

\section{Amour chevaleresque, amour galant et discours politiques de l'amour dans Les Plaisirs de l'île enchantée (1664)}

En 1647, Jean Chapelain, dans son dialogue De la lecture des vieux romans ${ }^{1}$, cherche à répondre à la question " de la galanterie des anciens chevaliers »: Lancelot était-il galant ? Tout en louant l'amour de Lancelot pour Guenièvre, Chapelain se refuse à faire de lui un galant : « Je ne le dirai pourtant pas, parce que je veux que la galanterie soit galante, et j'avoue que celle de Lancelot ne l'est $\operatorname{pas}^{2}$. » En quelques lignes Chapelain définit la galanterie comme un « art de plaire » et en bannit les chevaliers, véritables amoureux, mais dépourvus d'esprit et incapables « de se mettre bien auprès de [leurs] maîtresse[s] par des paroles étudiées $^{3} \gg$. Les chevaliers sont des amoureux qui ne savent pas parler d'amour, et c'est le discours qui fait le galant. La confrontation du modèle galant et du comportement des anciens chevaliers permet ainsi à Chapelain de construire l'idéal d'une conception de l'amour parfait qui unifierait les actes et le langage, l'éthique et l'esthétique.

Dès cette époque, et ensuite chez divers critiques, les rapprochements n'ont pourtant pas manqué pour affirmer la continuité des discours de l'amour, de la courtoisie des chevaliers à la galanterie des contemporains de Chapelain. Et les fêtes des Plaisirs de l'île enchantée, données par le Roi en 1664, semblent confirmer cette hypothèse. Fêtes galantes, elles mettent en scène des chevaliers, inspirés non du Lancelot mais du Roland furieux. Alors, ces chevaliers sont-ils galants ? Ont-ils appris à parler d'amour ? La figure du chevalier aurait-elle réalisé l'unification rêvée par Chapelain entre une éthique amoureuse et un langage, une esthétique ?

\footnotetext{
J. Chapelain, De la lecture des vieux romans, éd. J.-P. Cavaillé, Paris, Zanzibar, 1999. Ibid., p. 82. Ibid.
} 
Dans les textes qui rendent compte des Plaisirs de l'île enchantée on parle beaucoup d'amour ${ }^{4}$. Le livret distribué aux spectateurs au début des fêtes et la relation, plus longue, publiée après les fêtes pour être vendue et diffusée dans les cours étrangères, juxtaposent différents niveaux de récit : ils racontent parallèlement la fiction des fêtes et les conditions de leur représentation. On peut distinguer dans cet ensemble trois lieux du discours amoureux : le récit-cadre, les vers de Benserade insérés dans le récit de la course de bague de la première journée et les vers du président de Périgny à la louange de la jeune Reine, épouse de Louis XIV.

En juxtaposant ainsi références à la chevalerie et discours de l'amour, ces textes qui entourent les Plaisirs de l'île enchantée attestent non pas une survivance de la courtoisie médiévale mais la construction d'une éthique amoureuse moderne, associée à la célébration d'une politique et d'un ordre social eux aussi modernes.

\section{La galanterie : une éthique du plaisir ?}

\section{Amour et plaisir : l'île d'Alcine}

La relation des Plaisirs de l'île enchantée fait du plaisir le principe et le but des fêtes. Dès la première phrase, l'intention du Roi est de «donner aux Reynes, et à toute sa Cour le plaisir de quelques Festes peu communes ${ }^{5} »$. Cette intention de plaire est réciproque. La conclusion de la relation résume ainsi les fêtes : «Chacun a marqué si advantageusement son dessein de plaire au Roy, dans le temps où sa Majesté ne pensoit elle-mesme qu'à plaire ${ }^{6}$. » Les courtisans veulent plaire au Roi, qui lui-même cherche à plaire aux Reines. Si les plaisirs sont ainsi au centre des fêtes, c'est qu'ils manifestent la prospérité du royaume et la paix dont le Roi est le promoteur. Les plaisirs donnent à voir le pouvoir royal.

L'amour est au cœur des plaisirs. Très tôt, dès 1680, les fêtes des Plaisirs de l'île enchantée sont interprétées comme un discours amoureux, que le Roi aurait adressé non à la Reine mais à Mlle de La Vallière. C'était là une façon polémique de les considérer, dans la dynamique des récits hostiles à Louis XIV7, et il n'est pas

\footnotetext{
4 Je m'intéresserai particulièrement ici à deux de ces textes : le livret publié par Ballard avant la fête pour être distribué aux spectateurs, intitulé Les Plaisirs de l'isle enchantée, course de bague faite par le Roy, à Versailles, le 6 may 1664, et la relation publiée par le même Ballard dans les semaines suivantes, sous le titre Les Plaisirs de l'isle enchantée. Course de bague, Collation ornée de machines, Comedie meslée de Danse et de Musique, Ballet du Palais d'Alcine, Feu d'artifice: Et autres Festes galantes et magnifiques ; faites par le Roy à Versailles, le 7. May 1664. Et continuées plusieurs autres Jours.

5 Les Plaisirs de l'isle enchantée. Course de bague, Collation ornée de machines [...], Paris, Ballard, 1664, p. 3.

6 Ibid., n.p.

7 Cette interprétation, qui connaît une longue fortune critique, voit le jour dès 1680 dans un roman anonyme, Le Palais Royal ou les Amours de Mlle de la Vallière, publié à la suite de l'Histoire amoureuse des Gaules dans les Amours des dames illustres de notre siècle et histoire amoureuse des Gaules, Cologne, Jean Le Blanc, 1680. L'auteur applique le
} 
nécessaire d'avoir recours à ce type de lecture pour affirmer que le plaisir joue un rôle essentiel dans les fêtes et qu'il est lié, d'une manière ou d'une autre, au sentiment amoureux.

Cette omniprésente intention de plaire inscrit les fêtes dans une esthétique galante, revendiquée dès le titre de la relation : Les Plaisirs de l'isle enchantée. Course de bague, Collation ornée de machines, Comedie meslée de Danse et de Musique, Ballet du Palais d'Alcine, Feu d'artifice : Et autres Festes galantes et magnifiques ; faites par le Roy à Versailles, le 7. May 1664. Et continuées plusieurs autres Jours. Le plaisir est ainsi d'emblée mis en relation avec une morale amoureuse, l'éthique galante. Le récit-cadre, inspiré de l'Orlando furioso de l'Arioste, met en scène à travers l'épisode de l'île de l'enchanteresse Alcine différentes conceptions de l'amour, et oppose une éthique chevaleresque et galante à une érotique passionnelle. La magicienne Alcine retient les meilleurs chevaliers français prisonniers pour en faire ses amants. Le sujet est ainsi résumé au début du livret distribué aux spectateurs :

Les charmes d'Alcine, qui n'avoit pas moins de beauté que de sçavoir, retenant aupres d'elle, par un double enchantement, le brave Roger et plusieurs autres vaillants Chevalliers : toutes ses pensées ne s'occuperent plus qu'à empescher leur fuitte, pour faire durer ses plaisirs : Elle joignit à la force, et à la sçituation de son Palais, le pouvoir de ses Demons, la fierté de ses Geants, et celle de ses bestes farouches : Mais elle n'eust pas moins de confiance aux diverttissements des Promenades, de la Dance, des Tournois, des Festins, de la Comedie et de la Musique. Et comme elle avoit autant d'Amans que de Captifs, et qu'ils ne pensoient tous qu'à luy plaire : Ces Illustres Guerriers font une partie de Course de Bague ; et prenant pour sujet les Jeux Pythiens, ausquels Apollon presidoit, ils font leur entrée dans la Lice, avec tous les ornements dont ils peuvent l'accompagner, dans le plus beau lieu que la Nature et l'Art ayent jamais formé, et embelly pour le plaisir de la vie : Mais cette belle Magicienne, de qui les enchantements estoient d'une force prodigieuse, n'estant pas satisfaite que sa puissance parust en un seul endroit de la Terre ; afin de porter en tous lieux le triomphe de sa beauté, par les hommages de ces Chevalliers, a rendu son Isle flotante : Et apres avoir visité plusieurs Climats, elle la fait aborder en France, ou par le respect et l'admiration que luy causent les rares qualitez de la Reyne; Elle ordonne à ces Guerriers de faire, en faveur de sa Majesté, tout ce qu'ils auront peu inventer pour luy plaire par leur adresse, et par leur magnificence. ${ }^{8}$

schéma de La Princesse d'Élide, pièce de Molière jouée pendant les fêtes qui met en scène des princes amoureux donnant toutes sortes de divertissements à leur insensible maîtresse, à l'ensemble des Plaisirs de l'île enchantée. En faisant des fêtes un cadeau du Roi à sa maîtresse, il assimile la recherche galante du plaisir à la débauche. Ce texte relève de ce qu'Alain Viala appelle la " galanterie licencieuse » (La France galante, Paris, Puf, 2008, p. 203-212). En jouant sur l'ambiguïté du plaisir, il se donne comme un dévoilement de la face cachée du régime, dévoilement qui peut tourner à l'opposition politique déclarée.

Les Plaisirs de l'isle enchantée, course de bague faite par le Roy, à Versailles, le 6 may 1664, Paris, Ballard, 1664, « Première journée », p. 3-5. 
La relation qui lie les chevaliers à Alcine est bien amoureuse : ils sont ses « amants ». Mais pour Alcine, ils sont avant tout des objets de plaisir : elle veut les empêcher de fuir « pour faire durer ses plaisirs ». Et pour les retenir, elle leur procure à son tour des plaisirs : les « divertissements de la promenade, de la danse, des tournois, des festins, de la comédie et de la musique ». Ces plaisirs, qui concordent avec ce que propose Louis XIV à ses propres courtisans, instaurent une situation paradoxale. Le Roi apparaît comme un enchanteur, certes, mais Alcine est une enchanteresse malfaisante. Le bouquet final des fêtes dépasse cette homologie : un immense feu d'artifice donne à voir la destruction de l'île enchantée. Les chevaliers, lassés de leur prison, ont cherché à s'enfuir, et l'enchanteur Atlas est venu les secourir en donnant au chevalier Roger le moyen de rompre les enchantements d'Alcine.

Une condamnation du plaisir? Plaisir égoïste et magnificence

Faut-il en conclure que la fable des fêtes met en scène une condamnation de l'amour et du plaisir ? Ou bien qu'elle propose une métamorphose dans l'ordre des plaisirs et des valeurs qui leur sont associées?

Lorsqu'Alcine a le pressentiment de la destruction de son île, après s'être lamentée sur son malheur à venir elle envisage de demander du secours à la Reine mère, dont elle fait longuement l'éloge. Mais sa suivante repousse l'éventualité d'en obtenir de l'aide :
Je sçais qu'elle peut tout, mais je n'ose penser
Que jusqu'à nous deffendre on la vit s'abaisser.
De nos douces erreurs elle peut estre instruite,
Et rien n'est plus contraire à sa rare conduite ;
Son zele si connu pour le culte des Dieux
Doit rendre à sa vertu nos respects odieux,
Et loin qu'à son abord mon effroy diminuë,
Malgré-moy je le sens qui redouble à sa veuë.'

Les plaisirs d'Alcine sont ici condamnés au nom de la vertu. L'île enchantée est un lieu de péchés, de "douces erreurs ", odieux à la vertu royale. Comment concilier cette condamnation des plaisirs d'Alcine avec l'affirmation répétée du plaisir du Roi et des courtisans dans le récit des fêtes?

Le plaisir royal est inséré dans un échange fondé sur la réciprocité et la générosité, la magnificence, alors qu'Alcine est dominée par l'amour-propre et l'égoïsme. Son plaisir est fondé sur un amour non partagé. Alors que les chevaliers sont ses amants et cherchent donc à lui plaire, la Magicienne ne cherche qu'un plaisir égoïste. Au contraire, la générosité du plaisir royal est signalée par la

9 Ibid., « Ballet du Palais d'Alcine. Troisième Journée », p. 6. Ces vers sont du président de Périgny. 
magnificence des fêtes. Dès le titre de la relation, Les Plaisirs de l'île enchantée sont qualifiés de « fêtes galantes et magnifiques ». Ce dernier adjectif, qui renvoie à l'exhibition du don, est employé à huit reprises dans le récit pour qualifier les différents divertissements offerts par le Roi.

Le feu d'artifice final, en abolissant l'île d'Alcine comme espace des plaisirs, dépasse l'homologie entre Alcine et le Roi. Il existe donc dans le même spectacle des degrés de signification et de valeurs du plaisir, et le cheminement des trois journées qui le composent conduit à écarter les possibles déviances des plaisirs qui ne resteraient qu'au stade de l'illusion.

\section{Plaisir et libertinage}

À cette fin, la condamnation du libertinage est la condition nécessaire de la revendication du plaisir. Pour pouvoir faire du plaisir le principe des fêtes, il faut écarter la possibilité d'une interprétation en termes de vices, de libertinage. Cependant, le plaisir revendiqué dans la relation est loin d'être univoque. La fable d'Alcine permet de condamner - in extremis et dans les dernières pages - le vice et le libertinage. Mais à d'autres endroits du récit le plaisir n'est pas toujours présenté d'emblée comme vertueux. Ces fêtes prennent ainsi en charge un trait de la galanterie qu'Alain Viala a récemment souligné dans La France galante : la galanterie porte en elle une ambiguïté potentielle. Ainsi les vers de Benserade qui accompagnent les devises des chevaliers dans le récit de la course de bague de la première journée mettent en scène toute une gamme de conceptions de l'amour et du plaisir. Certains chevaliers revendiquent une conception du dévouement amoureux généreuse, aboutissement d'une conversion à la constance. Ainsi Ariodant (rôle tenu par le marquis d'Humières) :

Je tremble dans l'accés de l'amoureuse fiévre, Ailleurs sans vanité je ne tremblay jamais, Et ce charmant objet l'adorable GENEVRE, Est l'unique vainqueur à qui je me soûmets. ${ }^{10}$

Mais d'autres revendiquent l'inconstance. C'est le cas d'Astolphe (rôle tenu par le comte de Lude) :

De tous les Paladins qui sont dans l'Univers

Aucun n'a pour l'amour l'ame plus échaufée, Entreprenant toûjours mille projets divers,

Et toûjours enchanté par quelque jeune FÉE. ${ }^{11}$

Et le duc de Saint-Aignan, dans le rôle de Guidon le Sauvage, transforme même le discours amoureux en revendication de vigueur sexuelle.

10 Ibid., « Première journée », p. 14. Ces vers et les suivants sont de Benserade.

11 Ibid., « Première journée », p. 12. 
Les combats que j'ay faits en l'Isle dangereuse,

Quand de tant de Guerriers je demeuray vainqueur, Suivis d'une épreuve amoureuse,

Ont signalé ma force aussi bien que mon cœur. La vigueur qui fait mon estime,

Soit qu'elle embrasse un party legitime,

Où qu'elle vienne à s'eschapper ;

Fait dire, pour ma gloire, aux deux bouts de la Terre,

Qu'on n'en void point en toute guerre,

Ny plus souvent, ny mieux frapper.

POUR LE MESME.

Seul contre dix Guerriers, seul contre dix Pucelles

C'est avoir sur les bras deux étranges querelles,

Qui sort à son honneur de ce double combat

Doit estre ce me semble un terrible Soldat. ${ }^{12}$

Le personnage s'y prête : le chevalier Guidon le Sauvage est réputé pour avoir vaincu dix chevaliers dans un même combat avant de servir de mari à dix jeunes filles dans une même nuit. Il n'en reste pas moins que le plaisir amoureux mis en scène ici n'est pas tout à fait conforme à la plus stricte vertu. Cette équivocité du plaisir amoureux pose le problème de l'éthique qui va de pair avec l'esthétique galante.

\section{Discours de l'amour et fable politique : puissance, paix, soumission}

Les plaisirs de la paix

Un troisième lieu du discours amoureux dans le texte des Plaisirs, les vers du président de Périgny qui font l'éloge de la jeune Reine, épouse de Louis XIV, attribuent à Apollon les propos suivants :

J'ay toûjours pris plaisir à verser sur la France

De mes plus doux Rayons la benigne influence :

Mais le charmant objet qu'Hymen y fait regner,

Pour elle maintenant me fait tout desdaigner.

Depuis un si long-temps que pour le bien du monde

Je fais l'immense tour de la terre et de l'onde,

Jamais je n'ay rien veu si digne de mes feux,

Jamais un sang si noble, un cœur si genereux,

Jamais tant de lumiere avec tant d'innocence ;

Jamais tant de jeunesse avec tant de prudence ;

Jamais tant de grandeur avec tant de bonté ; 
Jamais tant de sagesse avec tant de beauté.

Mille Climats divers qu'on vit sous la puissance

De tous les demi-Dieux dont elle prit naissance,

Cedant à son merite autant qu'à leur devoir,

Se trouveront un jour unis sous son pouvoir.

Ce qu'eurent de grandeurs et la France et l'Espagne,

Les droicts de Charles-Quint, les droicts de Charle-Magne,

En elle, avec leur sang heureusement transmis,

Rendront tout l'Univers à son Trosne soûmis :

Mais un titre plus grand, un plus noble partage

Qui l'esleve plus haut, qui lui plaist davantage ;

Un nom qui tient en soy les plus grands noms unis,

C'est le nom glorieux d'Espouse de LOUIS. ${ }^{13}$

Un peu plus loin, un personnage de la suite d'Apollon, le Siècle d'Or, poursuit :

Si-tost qu'elle paroist dans cette heureuse terre,

Voy comme elle en bannit les fureurs de la guerre :

Comment depuis ce jour d'infatigables mains

Travaillent sans relâche au bon-heur des humains ;

Par quels secrets ressors un Heros se prepare

A chasser les horreurs d'un siecle si barbare,

Et me faire revivre avec tous les plaisirs,

Qui peuvent contenter les innocens desirs. ${ }^{14}$

Pour parler de la Reine, Apollon joue sur les métaphores : « Jamais je n'ai rien vu si digne de mes feux. » Une double relation amoureuse est mise en place : la Reine est désignée comme l'épouse du Roi et comme l'objet des feux d'Apollon ${ }^{15}$. La métaphore des " feux » amoureux est réactualisée par le rapprochement avec les rayons du Soleil, dans le cadre d'une mythologie louis-quatorzienne qui fait grand usage de l'image solaire.

Mais Apollon est aussi le dieu des arts, des plaisirs et de la paix. Le passage lie l'amour aux plaisirs et à la paix. Le mariage du Roi est figuré comme la source de la paix, ce qu'il est en pratique, en sa qualité de garantie du traité qui mit fin à la Guerre de Trente ans. Et la paix est le temps des plaisirs, " innocents » parce que mérités. À ces plaisirs innocents s'opposent les plaisirs coupables d'Alcine : elle a détourné les chevaliers de leur devoir, elle n'offre pas des plaisirs conquis mais des satisfactions illusoires. Alcine, aveuglée par ses plaisirs, mène son royaume à sa

13 Ibid., « Première journée », p. 17.

14 Ibid., « Première journée », p. 18.

15 Sur les discours qui entourent le mariage de Louis XIV et le rôle que joue la représentation de la Reine dans la construction de l'image du Roi, on se reportera aux analyses d'A. E. Zanger, Scenes from the marriage of Louis XIV, Stanford, Stanford University Press, 1997. 
perte. Au contraire, Louis XIV a offert à son royaume la paix et les plaisirs. On retrouve l'opposition entre un plaisir égoïste et un plaisir généreux, mais cette opposition est ici interprétée en termes politiques : la recherche du plaisir égoïste est dangereuse pour l'État, alors que celle du plaisir généreux en signale le bien-être et le bon gouvernement. Dans ce cadre, la fête des Plaisirs de l'île enchantée apparaît comme la revendication d'un succès politique : le mariage de Louis XIV a ramené la paix. Les divertissements offerts par le Roi sont le signe de cette paix, mais visent en même temps à affirmer que le Roi est attaché à la paix par désir du bien de tous, par amour pour ses sujets.

\section{L'amour comme fondement de la puissance politique}

Cependant, ce discours sur la paix s'accompagne d'une affirmation de puissance et d'une déclaration d'impérialisme. Le trône de France, héritier de l'empire de Charlemagne et de Charles Quint, est destiné à dominer toute l'Europe, voire le monde entier. Cette royauté universelle est présentée comme le moyen de faire régner partout la paix, et cette revendication de domination ne s'exprime justement pas en termes belliqueux. C'est ici le discours de l'Amour qui est utilisé pour dire la soumission de l'Europe à la Reine, et à travers elle au Roi.

\section{Mille climats divers qu'on vit sous la puissance De tous les demi-Dieux dont elle prit naissance, Cédant à son mérite autant qu'à leur devoir, Se trouveront un jour unis sous son pouvoir.}

C'est l'amour qui fait la soumission des sujets, et la Reine figure ainsi une sorte d'intermédiaire entre les sujets et le Roi : les sujets aiment la Reine, qui aime Louis. Le discours de l'amour est ainsi déplacé en discours de la soumission, au sein d'une fête dont la fonction première est une fonction de propagande politique. Car les ambitions françaises sont très concrètes : le mariage espagnol ouvre au trône de France la possibilité de revendiquer nombre de territoires en Europe, voire dans le monde.

Ce lien entre discours de l'amour et soumission politique éclaire d'un jour nouveau la description de l'île d'Alcine citée plus haut ${ }^{16}$. Le livret construit le récit cadre des fêtes comme une fable politique à deux degrés. Pour Alcine, les hommages que lui rendent les chevaliers constituent la manifestation d'une puissance qui vise à s'étendre de façon impérialiste :

Cette belle magicienne, $[\ldots]$ n'étant pas satisfaite que sa puissance parût en un seul endroit de la terre, afin de porter en tous lieux le triomphe de sa beauté, par les hommages de ces chevaliers, a rendu son île flottante. ${ }^{17}$

16 Voir supra.

17 Les Plaisirs de l'isle enchantée, course de bague faite par le Roy, à Versailles, le 6 may 1664, « Première journée », p. 4-5. 
L'amour est ici présenté comme le fondement de la puissance politique et la magnificence des fêtes et des divertissements est une exhibition de cette puissance. Parallèlement, le plaisir est désigné comme un outil d'assujettissement : c'est pour empêcher la fuite des chevaliers qu'Alcine multiplie leurs plaisirs.

Se construit ainsi un mode de discours par contrepoint : l'éloge royal se fera dans l'exhibition des qualités que les fêtes de Louis XIV atteignent et qui échappent à la Magicienne. La force du discours réside non dans une présentation en noir et blanc, mais dans la construction d'une gamme où chaque élément prend sens selon la relation qu'il entretient avec l'un des pôles.

\section{L'amour des fleurs de lys}

La comparaison du Roi et de la Magicienne est à la fois plus présente dans les textes et plus signifiante que l'identification de Louis XIV au chevalier Roger privilégiée par Jean-Marie Apostolidès ${ }^{18}$. Le vocabulaire du désir de plaire et de la magnificence, employé aussi bien à propos d'Alcine et de ses prisonniers que de Louis XIV et de sa cour, signale une application possible de la fable politique de l'île d'Alcine à la cour de Louis XIV. Mais ce qui distingue Louis XIV d'Alcine, c'est la légitimité de sa domination. Alcine utilise la soumission de ses chevaliers pour nourrir son plaisir égoïste : elle figure la tyrannie, qu'il est juste de renverser. $\mathrm{Au}$ contraire, la souveraineté de Louis XIV est fondée sur un désir de plaire réciproque, et c'est ce qui la rend légitime et juste.

Sa cour, comme celle d'Alcine, est une cour d'amants. Un seul exemple, celui des vers consacrés au prince de Marsillac, qui joue le rôle du chevalier Brandimart dans la course de bague :

Mes vœux seront contents, mes souhaits accomplis, Et ma bonne fortune à son comble arrivée Quand vous sçaurez mon zelle, aymable FLEURDELIS,

18 J.-M. Apostolidès, Le Roi-machine. Spectacle et politique au temps de Louis XIV, Paris, Éditions de Minuit, 1981, p. 98-101. J.-M. Apostolidès n'étudiant pas les textes du livret et de la relation comme des textes, mais comme des témoignages d'un spectacle, il prête peu d'attention au vocabulaire, aux reprises de termes ou d'expressions. Par contre, il construit son analyse à partir du fait que Louis XIV joue le rôle de Roger dans la course de bague, ce qui le conduit à ignorer les parallèles entre le Roi et Alcine, et à faire du personnage de Roger une représentation du Roi non seulement le temps de la course de bague, mais aussi dans la suite des fêtes, alors qu'il n'est plus joué par Louis XIV. Ce rapprochement le conduit à considérer l'ensemble des fêtes comme le moment où le Roi, tenté par le plaisir, réalise la nécessité de son dépassement et écarte définitivement le danger d'une recherche incontrôlée du plaisir qui nuirait à l'accomplissement de ses fonctions. Le plaisir serait donc convoqué pour mieux être écarté, au moins pour ce qui concerne le personnage du Roi. 
Au milieu de mon cœur profondément gravée. ${ }^{19}$

Fleur de Lys (Fiordiligi) est le nom, dans le Roland furieux, de l'épouse du chevalier Brandimart ; mais elle désigne aussi ici la fleur qui symbolise la monarchie française. Le discours amoureux est déplacé de la fiction-cadre au récit de la représentation, du chevalier au courtisan, et dans ce déplacement il se transforme en discours politique : l'amour de Brandimart pour Fleur de Lys, qui se traduit par le zèle, c'est-à-dire par la soumission et le service, devient amour du prince de Marsillac pour la France et soumission à celui qui incarne la nation, le Roi. Le discours amoureux fonctionne comme une image qui permet de dire la soumission, l'assujettissement, et qui les rend acceptables.

\section{La figure du chevalier galant}

Cette interprétation du discours amoureux comme discours de l'assujettissement permet de comprendre l'intérêt qu'il y a à mobiliser l'héritage des récits chevaleresques dans le cadre des fêtes : le personnage du chevalier galant devient une figure de la soumission de la noblesse.

\section{Le duc de Saint-Aignan dans le rôle de Guidon le Sauvage}

Le personnage de chevalier le plus fréquemment évoqué dans la relation des Plaisirs de l'île enchantée est celui de Guidon le Sauvage, représenté par le duc de Saint-Aignan. Non seulement il est nommé dans le récit de la course de bague et il y fait l'objet de deux poèmes différents ${ }^{20}$, mais il est encore repris à la fin du texte, dans un passage qui met en scène le duc de Saint-Aignan, et qui convoque à cette occasion le chevalier dont il a précédemment joué le rôle. Ce passage fait le récit d'un défi qui oppose le duc de Saint-Aignan et le marquis de Soyecourt. Dans le temps qui sépare le défi du combat, le duc écrit un quatrain adressé aux dames et dans lequel il utilise le personnage de Guidon le Sauvage :

Le Duc de $\mathrm{S}$. Aignan, avoit aussi fait voir à quelques uns de ses amis, comme un heureux presage de sa victoire, ces quatre Vers.

$$
\text { Aux Dames. }
$$

Belles, vous direz en ce jour,

Si vos sentimens sont les nostres,

Qu'estre vainqueur du grand Soyecourt

C'est estre vainqueur de dix autres.

Faisant toûjours allusion à son nom de Guidon le Sauvage, que l'avanture de l'Isle perilleuse rendit victorieux de dix Chevaliers. ${ }^{21}$

19 Les Plaisirs de l'isle enchantée, course de bague faite par le Roy, à Versailles, le 6 may 1664, «Première journée », p. 12.

20 Cités supra.

21 Les Plaisirs de l'isle enchantée. Course de bague, Collation ornée de machines, n.p. 
Le défi est finalement remporté par le duc, qui avait pourtant donné l'avantage à son adversaire. Ces deux passages ont pour point commun de valoriser les compétences guerrières du duc de Saint-Aignan, et de les mettre en relation, via le personnage de Guidon le Sauvage, avec un comportement amoureux : vigueur sexuelle dans le premier passage, galanterie dans le second avec le quatrain adressé « aux Dames ».

Il faut rapprocher cette image du duc écrivant des vers pour plaire aux dames d'une autre image du duc proposée par la relation : celle de l'inventeur du dessein des fêtes. En effet, dans l'introduction puis dans la conclusion de la relation, le duc de Saint-Aignan est présenté comme l'un des organisateurs des fêtes, celui qui en a inventé le sujet :

Monsieur de Vigarini, Gentilhomme Modenois, fort sçavant en toutes ces choses, inventa et proposa celles-cy ; et le Roy commanda au Duc de S. Aignan, qui se trouva lors en fonction de premier Gentilhomme de sa Chambre, et qui avoit déja donné plusieurs sujets de Ballet fort agreables; de faire un dessein ou elles fussent toutes comprises avec liaison et avec ordre ; de sorte qu'elles ne pouvoient manquer de bien reussir. Il prit pour sujet le Palais d'Alcine. ${ }^{22}$

Le poème de Saint-Aignan cherchant à plaire aux Dames est alors un redoublement du sujet des fêtes inventé par Saint-Aignan pour plaire au Roi.

\section{Le chevalier galant}

La figure du chevalier galant telle qu'elle se présente ici avec ce personnage de Guidon le Sauvage représenté par Saint-Aignan lie ensemble plusieurs composantes : la prouesse guerrière, la prouesse sexuelle ou le service galant des dames - on retrouve ici l'équivocité du plaisir amoureux -, le service du Roi et de ses plaisirs avec l'organisation des fêtes et une pratique d'écriture, aussi bien avec les vers galants destinés aux dames qu'avec les fêtes galantes destinées au Roi. Plus précisément, la guerre et l'amour sont liés dans cette figure de différentes façons. Par la prouesse, d'abord : la vertu guerrière est redoublée par la vigueur sexuelle. Mais aussi par le service du Roi : le service guerrier du Roi est déplacé en service amoureux.

En inventant Les Plaisirs de l'île enchantée Saint-Aignan cherche à plaire au Roi, et enfin par l'écriture, qui dit à la fois le service des dames et le service du Roi, le service guerrier et le service amoureux. La figure du chevalier galant réaliserait ainsi l'unification rêvée par Chapelain entre une éthique et une esthétique, tout en maintenant une ambiguïté essentielle dans la définition de cette éthique qui intègre des termes apparemment ou potentiellement contradictoires, à la fois dans l'ordre de l'amour et dans celui de la politique : du côté de la puissance, la prouesse guerrière et la vigueur sexuelle, et du côté de l'assujettissement, la soumission au Roi et la galanterie. L'ambiguïté de l'éthique amoureuse galante renverrait ainsi à

22 Ibid., p. 4. 
l'ambiguïté d'un discours politique capable d'affirmer dans un même mouvement la puissance guerrière de la noblesse et sa soumission au Roi.

La figure du chevalier galant permet ainsi de dire le service dû au Roi par la noblesse non pas en termes de devoir ou d'échange, mais en termes d'amour et de désir de plaire. Ce faisant, elle permet de placer sur le même plan différents types de service : le traditionnel service guerrier mais aussi, pourquoi pas, l'organisation des fêtes et des plaisirs du Roi. Les valeurs qui fondent la noblesse et sa place dans l'ordre social s'en trouvent modifiées. La vocation guerrière n'est plus ce qui définit la noblesse. Le regard du Roi, comme le regard de la maîtresse sur l'amant, est le seul capable de juger des mérites du noble, du plaisir que lui donne son service. Le déplacement du service guerrier dû dans le cadre d'obligations réciproques à des services diversifiés fondés sur le désir de plaire fait ainsi de la figure du chevalier une figure de la soumission de la noblesse.

Une figure de la domestication de la noblesse?

Les Plaisirs de l'île enchantée diffusent ainsi une idéologie nouvelle qui redéfinit les relations entre le Roi et la noblesse. Le discours de l'amour galant, parce qu'il est ambigu, permet de dire une soumission légitime de la noblesse au Roi fondée sur une réciprocité d'un type nouveau, le désir et le devoir de plaire.

Peut-on pour autant parler de domestication de la noblesse ? Le processus de domestication tel qu'il est défini par Norbert Elias ${ }^{23}$ suppose une éviction de la noblesse du pouvoir économique et militaire, au profit de la bourgeoisie. Dans ce modèle, les fêtes de cour sont la manifestation la plus visible de l'enfermement de la noblesse dans une logique de distinction par la dépense qui la ruine, et de son cantonnement dans des fonctions purement honorifiques, les charges de la maison du Roi.

La figure du chevalier galant laisse entrevoir une réalité toute autre. Elle permet à Saint-Aignan d'affirmer conjointement sa présence à la cour en qualité de premier gentilhomme de la chambre et sa puissance militaire (réelle, puisqu'il est lieutenant général des armées du Roi et qu'il sera bientôt gouverneur du Havre de Grâce) : les charges militaires et les charges de la maison du Roi, loin de s'exclure, se renforcent mutuellement. En outre, cette figure intègre des valeurs d'origine différente : les valeurs nobiliaires - le personnage du chevalier est une image traditionnelle du principe et des origines de la noblesse, il incarne l'ancienneté du second ordre et les valeurs qui le fondent, au premier rang desquelles la prouesse guerrière - et l'éthique galante, plutôt issue de milieux d'écrivains, proches de ce que Norbert Elias désigne sous le nom de bourgeoisie. Plus qu'une concurrence, elle manifeste donc un processus d'intégration des élites. Elle donne à voir une restructuration de la classe dominante et la construction d'une idéologie commune à une fraction de la noblesse et à des élites nouvelles.

23 Voir N. Elias, La Dynamique de l'Occident, Paris, Calmann-Lévy, 1976 ; La Société de cour, Paris, Calmann-Lévy, 1975. 
Les fêtes des Plaisirs de l'île enchantée ne manifestent donc pas le triomphe du monarque absolu sur une noblesse domestiquée mais la mise en place d'un ordre social nouveau, qui passe par la construction d'une idéologie intégrant valeurs nobiliaires et éthique galante. Dans ce cadre, les discours de l'amour permettent de dire la soumission et le pouvoir tout en les rendant acceptables. Les fêtes, et surtout les textes qui les entourent, jouent un rôle essentiel dans la diffusion de cette idéologie, voire dans la construction de son hégémonie.

Dans ce contexte, la mobilisation de l'imaginaire chevaleresque ne peut pas être interprétée comme le signe ou la trace d'une continuité qui mènerait de l'esthétique et de l'éthique médiévale de la courtoisie à la galanterie. Au contraire, c'est parce qu'il y a rupture avec l'idéologie féodale que le personnage du chevalier est convoqué, pour, dans un même mouvement, masquer et imposer l'idéologie nouvelle. Confondre courtoisie et galanterie, c'est se laisser prendre à cette construction idéologique.

Pour terminer par où j'ai commencé, revenons une dernière fois à Jean Chapelain et à son dialogue De la lecture des vieux romans. Lorsque Chapelain y considère la galanterie des chevaliers il en vient à rapporter les règles qui régissaient leurs comportements amoureux à une rationalité politique :

La politique de ces vieux temps fit passer en forme de la loi que les cœurs des dames seraient le prix du courage, aiguisant la fidélité que les vassaux devaient à leurs seigneurs par l'espérance, non seulement de la gloire mais encore du plaisir que produit la possession de la beauté. [...] Les mêmes princes, pour les mêmes fins, avaient mis dans l'esprit des dames que de toutes les vertus, la valeur était la seule considérable dans les chevaliers. ${ }^{24}$

En nous proposant cette lecture politique des discours amoureux du Lancelot, ne suggère-t-il pas que la galanterie, plus encore que la courtoisie, en même temps qu'elle est une esthétique et une éthique qui règle les rapports entre les sexes, est aussi un discours politique qui construit l'ordre social, une idéologie ?

C'est ce que montre l'étude des discours de l'amour dans les récits des Plaisirs de l'île enchantée. En construisant une homologie entre Louis XIV et la magicienne Alcine, Les Plaisirs de l'île enchantée confrontent une éthique galante et une érotique passionnelle et construisent un discours de l'amour ambigu, entre belle galanterie et galanterie libertine. Mais la confrontation d'Alcine et de Louis XIV est aussi une confrontation politique. Le plaisir d'Alcine est condamné parce qu'il est égoïste : Alcine utilise le désir de plaire des chevaliers à son profit, sans les payer de retour. Au contraire, la relation de Louis XIV et de ses courtisans est définie comme un désir de plaire réciproque, qui fonde la domination légitime. L'esthétique galante se fait non seulement éthique mais aussi politique.

24 J. Chapelain, op. cit., p. 75. 
$\mathrm{Au}$ double visage de l'amour galant répond alors l'ambiguïté de la politique galante, qui lie l'affirmation de la puissance de la noblesse et la revendication de sa soumission. Le discours de l'amour galant est déplacé sur le terrain politique et se fait discours sur la souveraineté et la domination légitimes. La mobilisation de récits chevaleresques utilise une figure traditionnelle de la noblesse pour construire une conception nouvelle de la relation qui lie le second Ordre au Roi. La formulation de cette relation en termes de service amoureux et de désir de plaire a pour conséquence une redéfinition du service légitime du Roi, qui dépasse le service guerrier. La construction de la figure du chevalier galant applique ainsi à la noblesse un principe de soumission à la monarchie fondé sur le désir de plaire. À travers cette figure complexe le discours de l'amour se fait discours de l'ordre social. Le service amoureux de la Dame dit que l'amour doit être une éthique du respect, non par déférence à la conjugalité catholique mais par choix personnel ; le danger des tentations libertines qui va de pair n'est pas ignoré, mais dépassé par l'affirmation du triomphe final des valeurs inscrites dans le choix des héros : l'hédonisme respectueux vaut alors comme signe d'adhésion, à la fois, à une morale amoureuse moderne et à la monarchie moderne, source de paix et donc de la possibilité même de cet hédonisme bien tempéré.

Marine Roussillon

Université de Paris 3 - Sorbonne Nouvelle Groupe de Recherches Interdisciplinaires sur l'Histoire du Littéraire (GRIHL) 